\title{
Changes in mortality patterns and temperature dependence of lifespan in Drosophila melanogaster caused by inbreeding
}

\author{
CJ Vermeulen and R Bijlsma \\ Department of Genetics, University of Groningen, Kerklaan 30, NL-9751 NN Haren, The Netherlands
}

\begin{abstract}
After an inbreeding event, lifespan can be curtailed through the expression of deleterious alleles. This will impact on both mortality patterns and interactions with the environment as visualised in reaction norms. We have established the effects of inbreeding on the temperature dependence of lifespan and on mortality patterns in Drosophila melanogaster. Four inbred lines displaying severely decreased lifespan and five outbred controls were assessed for male adult survival at three temperatures. As expected, all inbred lines showed a shorter lifespan than noninbred lines. The mechanisms behind this, however, appeared to be very diverse. Two inbred lines showed a significantly decreased temperature dependence of lifespan compared to the control lines. Analysis of variance on the mortality parameters over all lines showed that inbreeding changes the
\end{abstract}

age-independent mortality but not the age-dependent mortality, whereas temperature does the opposite. This suggests that gene-by-environment interaction caused by inbreeding is the result of changes in the processes of lifespan determination. Importantly, for the two other inbred lines, a particular temperature regime triggered the expression of conditional lethal alleles. Mortality was concentrated in short lethal phases early in adult life. These conditionally expressed lethal alleles affecting lifespan demonstrate line specificity for inbreeding depression and will help ageing studies as such alleles may serve as candidate genes for ageing processes and age-related pathologies in humans.

Heredity (2004) 92, 275-281, advance online publication, 17 December 2003; doi:10.1038/sj.hdy.6800412

Keywords: Drosophila; lifespan; ageing; temperature dependence; conditional lethals; inbreeding depression

\section{Introduction}

In recent years, interest has been kindled in the large influence that environmental factors can exert on the amount of inbreeding depression (Bijlsma et al, 1999; Fowler and Whitlock, 2002). Such issues are relevant to conservation biology, which deals with the fate of small populations in a rapidly changing environment, and also to research directed towards elucidating the genetic determination of life history characters. The actual causes of gene-by-environment interactions remain unknown for most characters, but may reflect significant changes in the underlying physiology and genetic determination of the affected traits. Lifespan appears to be a suitable trait for the study of such interactions, since it is assumed to be extremely sensitive to a myriad of environmental factors, ranging from temperature to food levels (Zwaan et al, 1991, 1992; Carey et al, 2002). Furthermore, lifespan can also become severely curtailed upon inbreeding, as has already been demonstrated by Clarke and Maynard Smith (1955), who showed that hybrids between two inbred lines showed a large increase in lifespan. It remains unresolved whether this involves changes in the ageing process or is caused by the emergence of novel determinants of lifespan, such as deleterious mutations in vital processes.

Correspondence: CJ Vermeulen, Department of Genetics, University of Groningen, Kerklaan 30, NL-9751 NN Haren, The Netherlands.

E-mail: CJVermeulen@biol.rug.nl

Received 14 October 2002; accepted 10 October 2003
In small inbred populations, survival can be drastically decreased as a result of inbreeding depression, through the expression of recessive deleterious alleles (Bijlsma et al, 1999, 2000; Armbruster et al, 2000; Keller and Waller, 2002). Inbreeding is known to lead to general decreases in various fitness measures, and also to very specific linespecific effects in Drosophila (Fowler and Whitlock, 2002) and in Tribolium (Pray and Goodnight, 1995). In a similar fashion, lifespan can be affected by inbreeding in these two different ways. Generally, inbred individuals are weak and thus extremely sensitive to environmental insults, due to a general decrease in fitness. This will decrease survival and lifespan under most, if not all, circumstances. On the other hand, lifespan can be affected by the expression of deleterious alleles with large effects (Simmons and Crow, 1977), whose expression is often conditional, depending on certain environmental factors such as ambient temperature. For this reason, inbreeding depression may become more pronounced under certain conditions, especially stress (Miller, 1994; Dahlgaard et al, 1995; Keller et al, 2002). Indeed, such environmental dependence of inbreeding depression has been shown for chromosome extraction lines (Bijlsma et al, 1999) and inbred vial populations (Bijlsma et al, 2000) of Drosophila melanogaster. Both of these mechanisms will become evident from the reaction norm for lifespan. Depending on the applied environmental factor, the shape of this reaction norm will be influenced in different ways. Lifespan in Drosophila is a temperature-dependent trait, and longevity is known to decrease exponentially with increasing temperature in 
the temperature range between 12 and $30^{\circ} \mathrm{C}$ (David, 1988). This presumably is caused by an increase in the rate of metabolic processes, which speeds up the ageing process. If, however, lifespan is limited by causes other than ageing, it will have different temperature dependence. Since environmentally induced mortality may be only very little affected by temperature, this means that severely inbred lines may display a reaction norm for lifespan and mortality patterns that are less affected by temperature. On the other hand, sudden temperaturedependent expression of deleterious alleles may lead to an exaggerated temperature response. The timing of the mortality due to these two mechanisms is radically different and should show up in an analysis of agespecific survival.

The aim of this research is to test the validity of the idea that inbreeding depression will decrease the temperature dependence of lifespan in D. melanogaster through an increase of overall mortality. We will show that general mechanisms indeed affect lifespan in this way, but that specific mechanisms can cause large line-specific temperature dependence. These specific mechanisms are particularly interesting, since these may provide information on possible changes in the genetic determination of lifespan upon inbreeding. To assess changes in the reaction norm of lifespan and mortality patterns with temperature, we measured longevity in males of inbred lines that displayed extreme lifespan phenotypes and in outbred lines at three temperature regimes and analysed the mortality data with life tables and mortality plots.

\section{Materials and methods}

\section{Stocks}

Inbred lines were established in 1997 at the same time as their outbred control lines. They were derived from the G83 base stock by seven generations of brother-sister mating (coefficient of inbreeding $F=0.785$ ). During the inbreeding procedure, selection was applied to fecundity and viability, to ensure that the inbred line could be maintained afterwards. This means that the lines were purged for most of the genetic load due to highly deleterious alleles. Since their establishment, these lines have been maintained in large numbers in halfpint bottles ( $40 \mathrm{ml}$ standard medium: $32 \mathrm{~g}$ dead yeast, $54 \mathrm{~g}$ sugar, $17 \mathrm{~g}$ agar and $13 \mathrm{ml}$ nipagine solution per litre) at $25^{\circ} \mathrm{C}$ and $40-60 \%$ relative humidity. Of both the inbred and outbred lines, 15 independently derived replicate lines were available, which are termed I 1-15 and $\mathrm{O} 1-15$, respectively. In 1998, mated longevity of these lines at 25 and $29^{\circ} \mathrm{C}$ was determined (Bijlsma et al, unpublished results). For the current experiment, we chose the five inbred lines on which inbreeding had the most marked effect on lifespan and took five lines at random from the outbred lines. These are I 1, 4, 7, 10 and 15 for the inbred lines and O 2, 6, 9, 12 and 13 for the outbred lines. The I1 inbred line showed unexpectedly high values for adult survival. Comparison to the phenotype of hybrid control flies and inspection of allozyme variation showed that this line was no longer inbred. Therefore, the I1 line was dropped from the analysis.

\section{Longevity assay}

Eggs were collected from an overnight egglaying session. These were transferred to half-pint bottles $(40 \mathrm{ml}$ standard medium + ampicillin $(100 \mathrm{mg} / \mathrm{l}))$ in noncrowding conditions (300 eggs/bottle). These bottles were transferred to an incubator $\left(25^{\circ} \mathrm{C} \pm 0.5\right)$ and flies were allowed to develop. Subsequently, male flies were collected over a 24 -h period using ice to immobilise the flies. In all, 60 flies for each line and temperature were distributed to vials with $7 \mathrm{ml}$ of standard medium with ampicillin (12 vials of five flies). Vials were transferred to incubators of 21,25 and $29^{\circ} \mathrm{C}$ $\left( \pm 0.5^{\circ} \mathrm{C}\right)$, and all were refreshed the next day. Thereafter, vials were refreshed twice per week for 21 and $25^{\circ} \mathrm{C}$, and three times per week for $29^{\circ} \mathrm{C}$. Dead flies were scored three times per week for each treatment. The experiment was performed in the period from March to June 2000.

\section{Data analysis}

Longevity of the flies was taken as the midpoint of the interval between two successive scorings in which the fly died. Flies that stuck to the medium but were still alive could not be transferred during refreshing of the vials. These flies were assumed to be dead at the next scoring. This assumption is valid, since sticking to the medium is due to weakening of the flies just before death. Flies that escaped or died because of accidents were dropped from analysis. Sample sizes were unequal due to these escapes and accidental deaths, but little error is expected because most escapes occurred before the onset of mortality, when flies were still young.

All data were analysed in SPSS (SPSS for windows, Release 9.0.1. SPSS Inc.) and in Statistix for windows (Version 1.0 Analytical software). Because variances of longevity were not homogeneous (Levene's test for homogeneity of variances) and because the distribution of lifespan is usually skewed to the left, longevity data were analysed with a nonparametric test (Kruskal-Wallis one-way analysis of variance by ranks).

Analysis of mortality patterns was performed using life tables. Cumulative survival rates for weekly intervals were constructed as described by Lee (1992).

To further characterise age-specific mortality, Gompertz plots were constructed. Weekly mortality was estimated using $\mu_{x}$ with

$$
\mu_{x}=-\ln \left(P_{x}\right)
$$

with $P_{x}$ as the weekly survival rate (Lee, 1992). Mortality $\mu_{x}$ is assumed to increase exponentially with age $x$, so

$$
\mu_{x}=\mu_{0} \mathrm{e}^{a x}
$$

with $\mu_{0}$ as the mortality at age 0 . The mortality parameters can be estimated by performing least-squares linear regression of $\ln \left(\mu_{x}\right)$ against age $x$, giving

$$
\ln \left(\mu_{x}\right)=\ln \left(\mu_{0}\right)+a x
$$

The intercept $\ln \left(\mu_{0}\right)$ is used as an estimate of ageindependent mortality and the slope $a$ as an estimate of age-dependent mortality (Lee, 1992).

Mean lifespan and estimates of the intercept and slope of the Gompertz plots were analysed with ANOVA using GLM procedure. All estimates were tested independently with inbreeding and temperature as the independent variables. 
Mortality parameters from lines I4 and I10 had to be corrected for premature mortality at certain temperature regimes, due to conditional lethal effects. This was accomplished by leaving out all mortality during the lethal phase (first 2 weeks for I4 and first week for I10). From these data, corrected mean lifespan and mortality patterns were estimated.

Temperature dependence of lifespan was estimated by linear regression of the reaction norm of median survival time (MST) against temperature. MST is derived by interpolation of $50 \%$ survival in the survival curves. The slope of the reaction norm represents the temperature dependence (in days $/{ }^{\circ} \mathrm{C}$ ) in this temperature interval. The data of the outbred control lines were used to construct a $95 \%$ confidence interval for this character. Thereafter, it could be established whether temperature dependence of the inbred lines was significantly different from control values.

\section{Results}

\section{Temperature dependence of lifespan}

Mean lifespan is given in Table 1 and survival curves are depicted in Figure 1. Conforming to expectations, inbred lines showed decreased adult survival compared to the outbred control lines. The Kruskal-Wallis test indicated large significant differences between lines $(P<0.001$ for all temperatures). Multiple comparison was performed to show differences between lines at the 0.05 significance level. The results of these are also shown in Table 1 . The variation in mean lifespan was very large in inbred lines, whereas control lines do not differ significantly from each other. This also becomes clear upon inspection of Figure 1, especially for the 21 and $29^{\circ} \mathrm{C}$ temperature regimes.

Reaction norms of MST against temperature are depicted in Figure 2a. MST is derived by interpolation of $50 \%$ survival in the survival curves and is used instead of mean survival to account for differences in the shape of the survival curves between lines (Figure 1). MST is lower at higher temperatures, and inbred lines usually have lower MST than control lines. To obtain relative changes in the reaction norms, relative survival of inbred lines compared to the mean of the outbred control lines was calculated (Figure 2b). Inbred lines I7, I10 and I15 appear to be relatively insensitive to temperature, as shown by the small absolute change in lifespan with temperature. Inbred line I4 on the other hand shows extreme temperature dependence with very low survival at high temperatures. Temperature dependence as judged by the slope of the reaction norm is significantly different from control values for all inbred lines.

Inspection of the survival curves and mortality plots of I4 and I10 reveals an interesting phenomenon. Both display a mortality peak in early adult life under a certain temperature regime. In inbred line I4, approximately $70 \%$ of the males died within the first 2 weeks after eclosion when kept at $29^{\circ} \mathrm{C}$. For the control lines, the mortality in this period was usually less than $10 \%$ (Figure 1c). At $21^{\circ} \mathrm{C}$ however, the survival curve of I4 males closely followed that of the control lines. Inbred line I10 was the mirror image of I4 in this respect, with $30 \%$ of the males dying in the first week when kept at $21^{\circ} \mathrm{C}$. The survival of the control lines in this period was
Table 1 Mean lifespan and Gompertz parameters for inbred lines and their outbred controls at 21,25 and $29^{\circ} \mathrm{C}$

\begin{tabular}{|c|c|c|c|}
\hline \multirow[t]{2}{*}{ Line } & \multirow[t]{2}{*}{ Lifespan } & \multicolumn{2}{|c|}{ Gompertz parameters } \\
\hline & & Intercept & Slope \\
\hline \multicolumn{4}{|l|}{$21^{\circ} \mathrm{C}$} \\
\hline O9 & $49.4^{\mathrm{a}}$ & -4.43 & 0.070 \\
\hline $\mathrm{O} 12$ & $50.0^{\mathrm{a}}$ & -3.17 & 0.038 \\
\hline $\mathrm{O} 2$ & $47.4^{\mathrm{a}}$ & -3.62 & 0.054 \\
\hline $\mathrm{O} 13$ & $44.1^{\mathrm{a}}$ & -3.14 & 0.048 \\
\hline O6 & $43.0^{\mathrm{a}}$ & -3.75 & 0.064 \\
\hline I4 & $41.0^{\mathrm{a}, \mathrm{b}}$ & -3.24 & 0.055 \\
\hline I15 & $32.6^{\mathrm{b}, \mathrm{c}}$ & -3.07 & 0.062 \\
\hline I10 & $27.3^{c}$ & $-3.21^{*}$ & $0.059^{*}$ \\
\hline I7 & $25.1^{\mathrm{c}}$ & -2.75 & 0.095 \\
\hline \multicolumn{4}{|l|}{$25^{\circ} \mathrm{C}$} \\
\hline O9 & $37.1^{\mathrm{a}}$ & -5.58 & 0.157 \\
\hline O6 & $35.6^{\mathrm{a}}$ & -3.71 & 0.086 \\
\hline O13 & $34.4^{\mathrm{a}, \mathrm{b}}$ & -4.03 & 0.099 \\
\hline $\mathrm{O} 2$ & $33.9^{\mathrm{a}, \mathrm{b}}$ & -3.83 & 0.095 \\
\hline O12 & $33.2^{\mathrm{a}, \mathrm{b}}$ & -2.81 & 0.062 \\
\hline I10 & $28.2^{\mathrm{b}}$ & $-3.26^{*}$ & $0.096^{*}$ \\
\hline I15 & $28.1^{\mathrm{b}}$ & -2.83 & 0.104 \\
\hline I7 & $20.1^{c}$ & -2.76 & 0.131 \\
\hline I4 & $19.8^{c}$ & $-1.71^{*}$ & $0.076^{*}$ \\
\hline \multicolumn{4}{|l|}{$29^{\circ} \mathrm{C}$} \\
\hline O6 & $28.3^{\mathrm{a}}$ & -4.10 & 0.162 \\
\hline $\mathrm{O} 13$ & $28.0^{\mathrm{a}}$ & -4.29 & 0.174 \\
\hline $\mathrm{O} 2$ & $26.4^{\mathrm{a}, \mathrm{b}}$ & -3.83 & 0.158 \\
\hline I10 & $26.5^{\mathrm{a}, \mathrm{b}}$ & -3.99 & 0.166 \\
\hline O12 & $26.2^{\mathrm{a}, \mathrm{b}}$ & -4.07 & 0.188 \\
\hline O9 & $26.2^{a, b}$ & -3.85 & 0.169 \\
\hline I15 & $22.5^{b, c}$ & -2.88 & 0.128 \\
\hline I7 & $18.8^{\mathrm{c}, \mathrm{d}}$ & -3.39 & 0.220 \\
\hline I4 & $11.3^{\mathrm{d}}$ & $-1.73^{*}$ & $0.130^{*}$ \\
\hline
\end{tabular}

Same letters in the lifespan data indicate homogeneous groups as indicated by the Kruskal-Wallis multiple comparison of mean ranks. Gompertz parameters estimated with data corrected for early adult lethality (see text) are marked with an asterisk.

usually $100 \%$ (Figure 1a). At $29^{\circ} \mathrm{C}$, the $\mathrm{I} 10$ males showed no such premature mortality. At $25^{\circ} \mathrm{C}$, both inbred lines expressed weak early adult mortality, showing an intermediate effect (Figure 1b). The temperature that triggers the lethal effect will be called the restrictive temperature (RT) and the temperature at which the lethal effect is absent the permissive temperature (PT), after the terminology used by Suzuki (1970). Comparison of the reaction norms of these two lines to the other inbred lines may be required, since their survival may depend on very different mechanisms, but could not be reliably performed, as the sample size for the control inbred lines would become too small $(n=2)$.

\section{Patterns of mortality}

Mortality plots can reveal patterns in age-specific mortality (Lee, 1992). In an outbred population, mortality plots normally show an exponential increase that is characteristic of the ageing process. Inbreeding is expected to change the shape of the mortality curve. The increase in mortality is expected to be lower with inbreeding if 'normal' ageing becomes unimportant for longevity. In the case of acceleration of the ageing process, this increase will become steeper. Another 


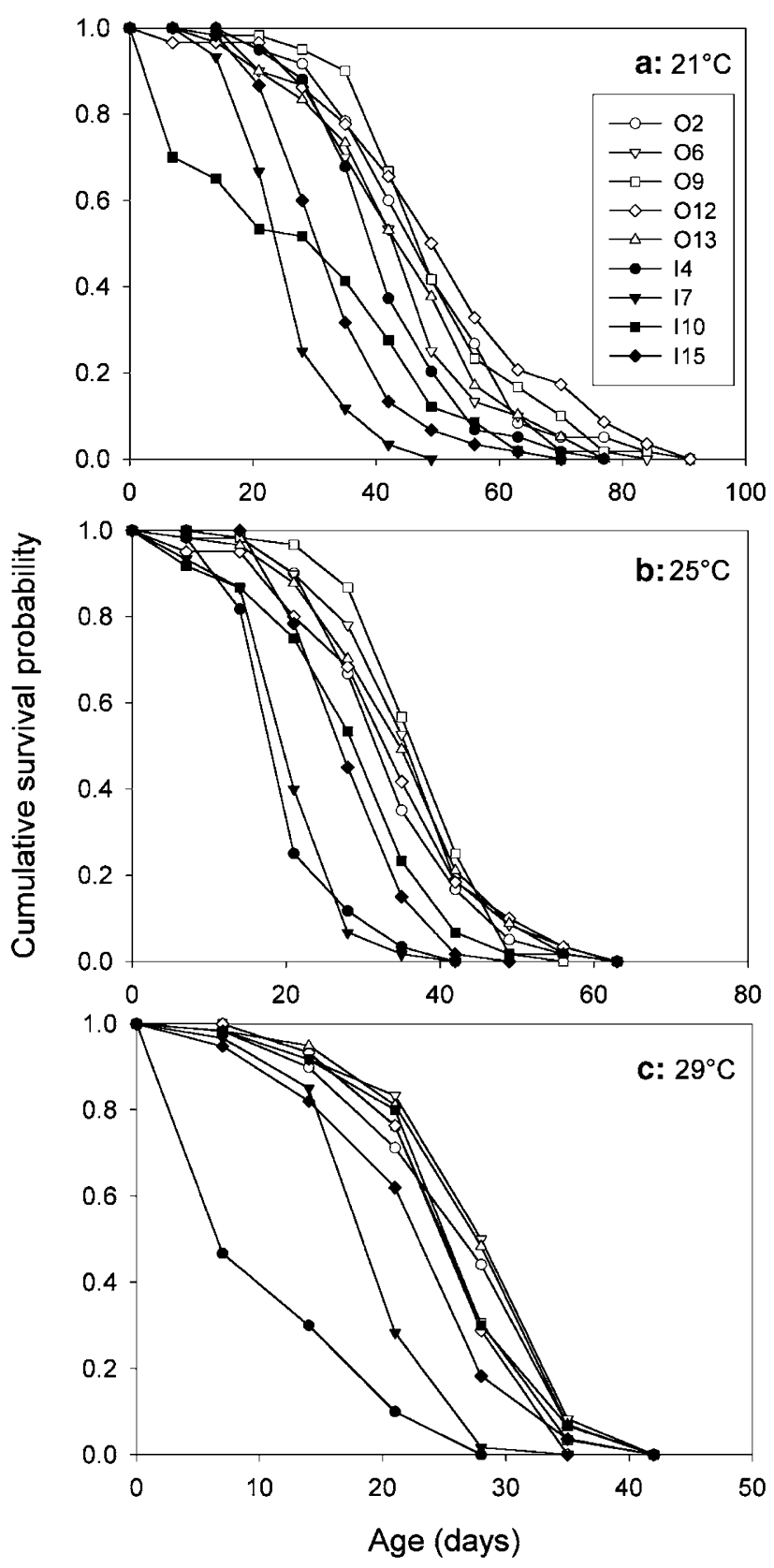

Figure 1 Cumulative survival probability as a function of age for inbred (closed symbols) and control lines (open symbols) at $21^{\circ} \mathrm{C}$ (a), $25^{\circ} \mathrm{C}$ (b) and $29^{\circ} \mathrm{C}($ c), respectively.

possibility is the occurrence of sudden 'mortality peaks' as certain genetic diseases strike.

The mortality plots unambiguously showed that all inbred lines show an increase in age-specific mortality at all temperatures (data not shown). This increase is the hallmark of ageing, although it should be noted that the exact underlying mechanism may be totally different from that in the outbred controls in these lines. In the Gompertz plots, regression of age-specific mortality against age was significant at the 0.05 level for all lines. The only exception was the I4 line that gave a nonsignificant increase at 25 and $29^{\circ} \mathrm{C}$. This is caused by the lack of data points for this line, due to its high rate of early mortality (see Figure 1c) rather than by the slope of regression itself.
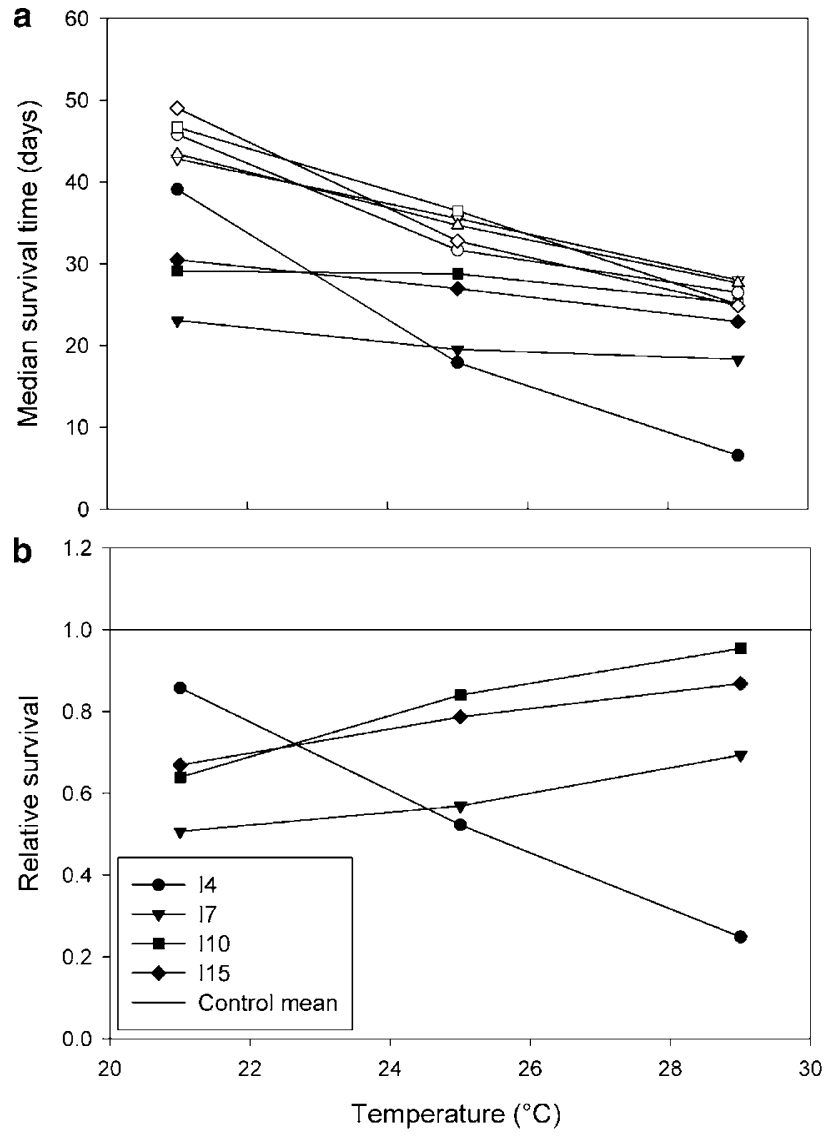

Figure 2 Reaction norms of MST (a) and relative survival (b) against temperature. Median survival is derived by interpolation of $50 \%$ survival in the survival curves. Relative survival of the inbred lines is derived by scaling the mean MST of all control lines to 1 (reference line).

Inbreeding affected the mortality in two ways in this experiment. Lines I4 and I10 showed very specific patterns at their respective restrictive temperatures (Figure 1). Large mortality peaks occurred early in adult life as the lethal phase of these lines set in. These demonstrate line-specific patterns of mortality. More general effects of inbreeding on mortality are shown by the analysis of variance. Mean lifespan and mortality patterns were separately analysed by ANOVA using inbreeding and temperature as independent variables (Table 2). The mortality parameters of lines I4 and I10 were corrected for premature mortality at the RT as described in Materials and methods. Both inbreeding and temperature significantly affect lifespan, but there is no significant interaction. Inbreeding and temperature appear to have different effects on the mortality curves. Inbreeding significantly affects age-independent mortality but not the slope. Temperature on the other hand affects the slope but not the intercept. The fact that the null hypothesis is rejected at such low sample size indicates that these effects are indeed very large.

\section{Discussion}

In a simplified model we can regard ageing as being determined by two major components. On the one hand, there is an internal deterioration that depends on metabolic processes and therefore is temperature sensitive. 
Table 2 ANOVA using GLM procedure for mean lifespan and the Gompertz parameters with temperature and inbreeding as independent factors

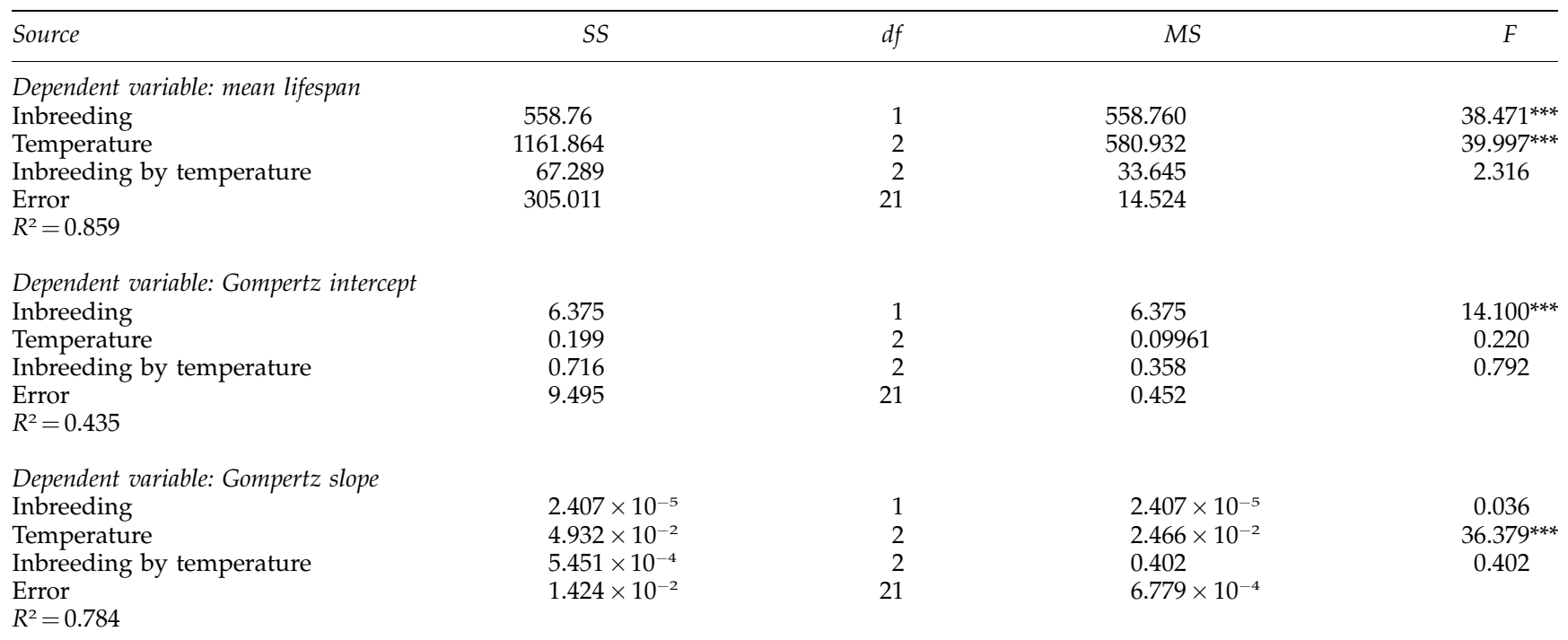

$* * * P<0.001$.

On the other hand, there is a defence against external insults such as infection and this is expected to be relatively temperature insensitive, because it presumably depends less on enzyme kinetics and metabolic reactions. In inbred lines, the latter may have an increased impact due to the overall decrease in performance known as inbreeding depression. In this study, two inbred lines (I7 and I15) show a significantly decreased response to temperature (Figure 2). This is possibly due to a decreased impact of metabolic processes normally limiting for lifespan, that is, 'normal' ageing. We think that the flies of these lines are more sensitive to environmental insults and thus lifespan of these lines will be relatively temperature insensitive. The inbreeding effect was also evident from the mortality plots. It has often been pointed out that mortality plots can only be reliably constructed from assays of a sufficient number of individuals (eg Khazaeli et al, 1996; Pletcher and Curtsinger, 1998), as estimates for late-life mortality become inaccurate through sampling error. As sample size in this experiment is small, caution must be taken in interpretation of the results. Nevertheless, effects of inbreeding on mortality patterns were often very large and could easily be identified by using mortality plots on these data. Interpretation of the ANOVA shows that inbred lines experience a higher overall mortality, as shown by a higher age-independent mortality. This supports the idea that flies of these lines have increased mortality at all ages, and that their decreased lifespan is the result of an overall weakness, rather than an acceleration of the ageing process. Still, both mortality parameters have been found to be affected under selection for lifespan (Nusbaum et al, 1996; Zwaan, 1999), even though traditionally the slope is viewed as the 'rate of ageing'. For example, if judged solely by the Gompertz slope, the I7 line can be said to experience accelerated ageing (Table 1). Nevertheless, we believe that radically different processes might be responsible for lifespan in all inbred lines. The alternative is that there is an interaction that does lead to an increase in the ageing process, but this would not decrease the temperature dependence. According to the ANOVA, temperature affects the rate of ageing, which is consistent with the concept that temperature affects metabolic rate (David, 1988). Nevertheless, there seemed to be no gene-by-environment interaction, as shown by the nonsignificance of the inbreeding by temperature interaction for lifespan. This is presumably caused by the extreme temperature sensitivity of the I4 inbred line (even after correction), which cancels the insensitivity of the other inbred lines. The analysis of variance has been carried out with low sample size (four inbred lines and five control lines). This calls for caution in the interpretation of the results, all the more because two lines had to be corrected for premature mortality.

As opposed to these general effects of inbreeding, the experiment also showed very specific lethality in two inbred lines (I4 and I10). These lines showed expression of temperature-sensitive mortality. This mortality occurs during a narrow time window in early adult life. Presumably, in these lines, fixation has occurred of conditional lethal alleles, conceivably involving only a single locus in each respective line. The flies die because they are suffering from a genetic disorder. If the lethal alleles occur in very low frequencies in natural populations, variation at the genes responsible presumably has little or no effect on lifespan in outbred populations. Temperature-sensitive (ts) conditional lethal alleles have been described quite often in the literature and several are known to affect adult survival (Baird and Liszczynskyj, 1985; Homyk et al, 1986). In addition, ts mutations affecting lifespan and ageing are known from other model organisms such as Saccharomyces cerevisiae and Caenorhabditis elegans (eg Gems et al, 1998). All these studies concerned mutants that were induced by mutagenesis. The lethals in this study are either naturally occurring alleles or have spontaneously arisen in the laboratory. Such a ts adult lethal has been found before in the G83 base stock (Oudman, 1991). Mutants can be expected to occur in low frequencies in any population 
(Simmons and Crow, 1977), and if these are conditional they might rise to considerable levels at permissive conditions, especially under genetic drift. The existence of conditionally deleterious alleles probably is an explanation for the extreme dependence on the environment that might occur in inbred populations and for the increased extinction probability of such populations (Bijlsma et al, 1999).

The occurrence of alleles causing strong gene-byenvironment interaction in the G83 base population (and presumably also in the natural population from which it is derived) suggests that inbreeding depression will be very sensitive to environmental fluctuations, at least in some inbred lines. These type of alleles may be responsible for the large dependence on the environment of QTLs responsible for lifespan differences between two inbred lines of Drosophila in the QTL mapping study of Leips and Mackay (2000). Several studies have confirmed that inbreeding depression is more pronounced in a stress environment (Bijlsma et al, 2000 and references therein). Other studies however failed to find this (Armbruster et al, 2000; Fowler and Whitlock, 2002).

Large effect mutants indicate genes potentially harboring genetic variation for the affected trait. This means that the conditional lethals are of potential interest for research on the genetic determination of lifespan and ageing. Research on major genes affecting bristle number in Drosophila showed that several loci formerly known to have large bristle mutant phenotypes also segregated variation in natural populations (Mackay, 1995). But despite the convenience of large effect mutants, extreme caution should be taken when interpolating the results of lifespan mutants to 'normal' ageing. Unlike bristle number, lifespan is a trait that can be affected by many other genes that are not causually involved in lifespan differences in outbred populations, but disrupt general homeostasis. In organisms displaying decreased survival due to genetic disorders, different physiological processes than those normally involved in ageing may become limiting for lifespan.

The inbred lines in this study were chosen from a group of 15 replicates because of their low adult survival. The use of lines that were known to have large reductions in adult survival will have exaggerated the inbreeding effect and almost certainly has enlarged the chance of finding lethals. In corroboration of our results, Hughes (1995) found significant inbreeding depression for male adult lifespan in lines homozygous for the third chromosome, showing that this character can indeed be subject to inbreeding depression. In addition, it has been several years since our lines were established, so purging is expected to have taken place since, declining the initial inbreeding depression in these lines. Fowler and Whitlock (1999) showed such recovery of fitness in inbred lines of Drosophila. However, the use of lines with known decreases in longevity a year after establishment ensured that the inbred lines were fixed for alleles with major effects. Furthermore, because of the high degree of inbreeding in the lines used in these experiments, a complete fitness rebound is not expected. Such long-term effects of inbreeding have been shown to persist in similarly derived lines of D. melanogaster (Bijlsma et al, 2000).

In this study, we have shown that there is a general effect of inbreeding on lifespan that increases overall mortality and decreases the temperature dependence of this trait. Particularly interesting is the observation that there are also several instances of specific conditionally expressed lethal effects. These results are of interest to conservation biology that deals with gene-by-environment interactions in small inbred populations. Furthermore, the study of natural mutants affecting lifespan in inbred populations may contribute to a better understanding of genetic variation for longevity in model organisms and may also be relevant as model systems for pathologies in humans.

\section{Acknowledgements}

We thank Wilke van Delden and Bas Zwaan for their valuable comments on early versions of the manuscript, and Anneke Boerema for taking care of the experiment during the absence of the first author. Also, the comments of two anonymous reviewers greatly helped to improve the manuscript.

\section{References}

Armbruster P, Hutchinson RA, Linvell T (2000). Equivalent inbreeding depression under laboratory and field conditions in a tree-hole-breeding mosquito. Proc $R$ Soc Lond $B$ 267: 1939-1945.

Baird MB, Liszczynskyj J (1985). Genetic control of adult lifespan in Drosophila melanogaster. Exp Gerontol 20: 171-177.

Bijlsma R, Bundgaard J, Van Putten WF (1999). Environmental dependence of inbreeding depression and purging in Drosophila melanogaster. J Evol Biol 12: 1125-1137.

Bijlsma R, Bundgaard J, Boerema AC (2000). Does inbreeding affect the extinction risk of small populations? Predictions from Drosophila. J Evol Biol 13: 502-514.

Carey JR, Liedo P, Harshman L, Liu X, Muller HG, Partridge L et al (2002). Food pulses increase longevity and induce cyclical egg production in Mediterranean fruit flies. Funct Ecol 16: 313-325.

Clarke JM, Maynard Smith J (1955). The genetics and cytology of Drosophila subobscura XI. Hybrid vigour and longevity. J Genet 53: 172-180.

Dahlgaard J, Krebs RA, Loeschcke V (1995). Heat-shock tolerance and inbreeding in Drosophila buzattii. Heredity 74: 157-163.

David JR (1988). Temperature. In: Lints FH, Soliman MH (eds) Drosophila as a Model Organism for Ageing Studies, Blackie: London pp 33-45.

Fowler K, Whitlock MC (1999). The variance in inbreeding depression and the recovery of fitness in bottlenecked populations. Proc $R$ Soc Lond B 266: 2061-2066.

Fowler K, Whitlock MC (2002). Environmental stress, inbreeding, and the nature of phenotypic and genetic variance in Drosophila melanogaster. Proc $R$ Soc Lond B 269: 677-683.

Gems D, Sutton AJ, Sundermeyer ML, Albert PS, King KV, Edgley ML et al (1998). Two pleiotropic classes of daf-2 mutation affect larval arrest, adult behavior, reproduction and longevity in Caenorhabditis elegans. Genetics 150: 129-155.

Homyk Jr T, Sinclair DAR, Wong DTL, Grigliatti TA (1986). Recovery and characterization of temperature-sensitive mutations affecting adult viability in Drosophila melanogaster. Genetics 113: 367-389.

Hughes KA (1995). The inbreeding decline and average dominance of genes affecting male life-history characters in Drosophila melanogaster. Genet Res 65: 41-52.

Keller LF, Grant PR, Grant BR, Petren K (2002). Environmental conditions affect the magnitude of inbreeding depression in survival of Darwin's finches. Evolution 56: 1229-1239. 
Keller LF, Waller DM (2002). Inbreeding effects in wild populations. TREE 17: 230-241.

Khazaeli AA, Xiu L, Curtsinger JW (1996). Effect of density on age-specific mortality in Drosophila: a density supplementation experiment. Genetica 98: 21-31.

Lee ET (1992). Statistical Methods for Survival Data Analysis, 2nd edn. Wiley: New York.

Leips J, Mackay TFC (2000). Quantitative trait loci for life span in Drosophila melanogaster: interactions with genetic background and larval density. Genetics 155: 1773-1788.

Mackay TFC (1995). The genetic basis of quantitative variation: number of sensory bristles of Drosophila melanogaster as a model system. Trends Genet 11: 464-470.

Miller PS (1994). Is inbreeding more severe in a stressful environment? Zoo Biol 13: 195-208.

Nusbaum TH, Mueller LD, Rose MR (1996). Evolutionary patterns among measures of ageing. Exp Gerontol 31: 507-516.

Oudman L (1991). A locus in Drosophila melanogaster affecting heat resistance. Hereditas 114: 285-287.
Pletcher SD, Curtsinger JW (1998). Mortality plateaus and the evolution of senescence: why are old-age mortality rates so low? Evolution 52: 454-464.

Pray LA, Goodnight CJ (1995). Genetic variation in inbreeding depression in the red flour beetle Tribolium castaneum. Evolution 49: 176-188.

Simmons MJ, Crow JF (1977). Mutations affecting fitness in Drosophila populations. Annu Rev Genet 11: 49-78.

Suzuki DT (1970). Temperature-sensitive mutations in Drosophila melanogaster. Science 170: 695-706.

Zwaan BJ, Bijlsma R, Hoekstra RF (1991). On the developmental theory of ageing. I. Starvation resistance and longevity in Drosophila melanogaster in relation to pre-adult breeding conditions. Heredity 66: 29-39.

Zwaan BJ, Bijlsma R, Hoekstra RF (1992). On the developmental theory of ageing. II. The effect of developmental temperature on longevity in relation to adult body size in Drosophila melanogaster. Heredity 68: 123-130.

Zwaan BJ (1999). The evolutionary genetics of ageing and longevity. Heredity 82: 589-597. 